\title{
OPERATING SYSTEM VIRTUALIZATION IN THE EDUCATION OF COMPUTER SCIENCE STUDENTS
}

\author{
Andrej Cvetkovski \\ Assoc. Prof. Dr., Mother Teresa University, North Macedonia, acvetk@gmail.com
}

\begin{abstract}
Virtualization is a way of simultaneously running multiple operating systems on a physical computer. Virtualization can run on virtually every machine, from embedded systems-on-chip, to large data centers and cloud environments. Simple on its surface, virtualization taps into possibilities that would be either unavailable or too involved to obtain from non-virtualized hardware. In this light, the aim of this paper is to provide a survey and analysis of virtualization and its applications, and trace ways of its usage in teaching of CS courses as well as the equipping of well-rounded CS laboratories with virtualization capabilities. The hope is that this survey will be of value to instructors, laboratory administrators as well as to advanced students undertaking a self-study of operating systems and their applications.
\end{abstract}

Keywords: Multiple Operating Systems, Virtualization, Datacenter, Cloud, Computer Science Education.

\section{INTRODUCTION}

Virtualization is a way of running virtual computer systems on a single physical computer. At present, virtualization is a ripe technique supported in the major CPU designs as well as the necessary virtualization software, and is already an inextricable part of production environments. With that, computer virtualization ought to become an inextricable part of the computer science (CS) education as well. Virtualization is useful because it provides several possibilities in addition to classical in-hardware operation: running multiple operating systems simultaneously; easier software installations; testing and disaster recovery; infrastructure consolidation, to name but a few.

As far as the fundamental virtualization technologies are concerned, there are three major virtualization approaches (Kolyshkin, 2006): emulation, paravirtualization and operating system-level virtualization. In the emulation paradigm, operating systems that support the underlying platform are being emulated, either by emulating the processor capabilities, or by passing code to the physical CPU on the host system. The emulated environments are usually of low performance compared to other approaches. Para-virtualization on the other hand is run on a hypervisor - also known as a monitor - which represents a thin layer between the host and the guest operating system. While the performance of para-virtualization is generally better than that of emulation, it has the disadvantage that it requires modifications to the guest operating systems in order for them to operate properly. Finally, the operating system-level virtualization enables kernel virtualization and therefore it is possible to run multiple isolated environments in parallel on a single host operating system. In all cases, the use of virtualization provides a virtual environment also known as a virtual container, which is an isolated program execution environment with its own set of processes, independent of other, possibly coexisting machines on the same physical server. This gives rise to possibilities, such as for example: improved server consolidation, allowing better organization in the server room, reducing the number of physical servers and the physical operating systems running on them. Another advantage is that security can be improved dramatically via isolated virtual environments. In addition, there is a possibility to perform the so-called teleportation that is live migration of the installations. Thus, with operating system level virtualization we talk of operating system hosting, where users of virtual environments have full set of administrative privileges on the hardware and even communication devices to which the container is 
interfaced. This allows much simplified software development and testing, and therefore plays a very significant role in the education of computer science students. With such setups, each student can have a virtual environment, which can be re-created, duplicated or destroyed within minutes, unlike the equipment in the classical computer laboratory.

In the following, we will focus on OS-level virtualization and will be using the current standard terminology: Host operating system (host) is an operating system running on physical computing hardware. Guest operating systems (guest) are virtual installations running atop the host operating systems. A Virtual machine (VM) represents a container of data and metadata allowing together a guest OS to run in a shared environment.

A non-negligible number of courses in the curricula of computer science programs can benefit from the use of operating system virtualization in computer laboratories, as well as the conduct of lectures, exercises and completing homework assignments. Consequently, the virtualization of operating system has found its applications in numerous teaching areas such as teaching of operating system administration and architecture; distance education; moving information technologies for education to cloud computing; energyefficient computing; the use of thin clients; applications of virtualization in forensics; teaching of communication and computer network design and administration. Virtualization technology, however, given its intrinsic complexity and the gamut of possibilities it offers, presents a number of challenges to be overcome by higher education instructors, lab administrators, students, etc.

In this light, the aim of this paper is to provide a survey and analysis of virtualization and its applications, and trace the ways of its use in teaching of CS subjects as well as the equipping well-rounded CS laboratories with virtualization capabilities. The hope is that this survey will be of value to instructors, laboratory administrators as well as to advanced students undertaking a self-study of operating systems and their applications.

\section{STATE-OF-THE-ART FEATURES IN VIRTUALIZATION}

The provisions for portability, export and import of virtual machines today, coupled with the fast SSD storage devices enable VMs to be created, cloned, replicated or deleted within seconds. Effectively, one has a structure of branched snapshots and cloning, allowing for storage of VM's state in a dynamic tree-structure.

Guest additions represent modifications of the guest operating systems, allowing shared folders between the host and the guests, seamless windows integration in a graphical user interface environment, 3D acceleration of graphics etc.

On the hardware support side, notable state-of-the-art features offered by today's virtualization solutions are: guest multiprocessing (SMP) - where guest operating systems have direct access to the cores or threads of the physical CPU; support for transparently connecting physical USB devices to the guest OSes; full ACPI support, allowing guest operating systems to take advantage of the reduced-power CPU C-states, multiscreen resolutions, PXE Network boot etc.

Virtual storage devices have an impressive support in today's virtualization technology. All common controllers are presented to the virtual OS. Examples include IDE, SATA (AHCI), SCSI, SAS, USB MSD, and NVMe. Disk image files are the norm in the use of guest VMs, and several standard formats are supported, such as VDI, VMDK, VHD, and HDD. Virtual storage is supported by features such as differencing images, cloning disk images, CD/DVD support.

Virtual networking. Virtual networking is supported by virtualizing networking hardware in several networking modes such as network address translation (NAT), bridged networking (intended to share the physical interfaces of the host hardware with the guest installations), internal networking (limited to guest OSes), host-only networking, UDP tunnel networking, virtual distributed Ethernet (VDE) etc.

Remote use of virtual machines is also supported by a range of impressive features, including remote display (VRDP) support, running of headless guests, remote desktop servers, remote USB connection, RDP authentication, RDP encryption, multiple connections to the VRDP server, multiple remote monitors, VRDP video redirection etc.

Teleporting is the process of moving a virtual machine over a network from one physical host to another, while the virtual machine is running. Teleporting plays an important role in high-availability servers, and is supported in several major implementations of OS-level virtualization.

Scripting for VM Management allows the administrator of a virtualized environment to control virtual machines from the command line of the host operating system. This includes creating, modifying, and cloning of VMs, import and export of VMs, full control while running a VM, and gathering metrics and 
statistics about the operation of the VMs.

Other advanced features in the state of art virtualization platforms include CPU hot-plugging, $\mathrm{PCI}$ passthrough, raw host hard disk and access from the virtual machines to physical iSCSI servers, etc.

Finally, heightened security in a virtualized environment is achieved by encapsulating a guest and executing it in a protected environment, as well as encryption of RDP (if used) and encryption of virtual storage.

\section{OPERATING SYSTEM VIRTUALIZATION IN THE EDUCATION OF COMPUTER SCIENCE STUDENTS}

A number of authors have recently pointed out the advantages of operating system virtualization in the education of computer science students (see the List of References). Gaspar et al. (2008a, 2008b, 2008c) review the past, present, and future in the adoption of virtualization. Agrawal et al (2014) report the implementation of virtualization on thin client/server architecture as an application of green computing. While consolidation through virtualization on the server side is not new technology, recently with the rise of workstation and desktop computing power, we can talk about consolidation at the client (particularly desktop) side as a natural consequence of the advances in computing technology.

Sayler et al. (2014) discuss that increasingly, students bring their own devices such as portable computers, laptops, tablets etc. to the computer laboratory or the CS classroom. Therefore, the hardware environment is increasingly diverse and poses a challenge to distributing a uniform set of development environments for the core CS courses. However, with the virtualization at hand, it is perfectly possible to deploy a uniform set of tools while employing a limited number of system lab administrators. Therefore, virtualization is a natural enabler of the "bring your own device" (BYOD) concept of computer science laboratory, and it is especially effective in educational institutions with budget limitations located in less developed countries.

The computing cloud is a relatively new technology, allowing access to a set of remote servers through the publicly routed Internet in order to access data and applications (Bala, 2010). The cloud, by its nature, represents virtualization of resources while hiding the complexity of its management and maintenance from its end users. By using virtual technology, an educational institution can become (1) an infrastructure provider, providing infrastructure as service which can be used on demand to run applications operating systems and services for the organization or individual department needs, while providing at the same time both local and global access transparently; (2) a provider of platform services, providing consolidated access to security-enhanced OS platforms on premises; and (3) a provider of software services.

A thin client is an end-user terminal which is designed to connect to other, computationally more capable devices such as workstations or servers, and take the advantage of their higher computing power without the need to duplicate hardware, thus reducing costs, system complexity, energy expenditures, and administration efforts (Cimen et al., 2014). In recent years, the use of thin clients and powerful servers becomes especially interesting in the context of operating system virtualization. Cimen et al. (2014) presented their testbed configuration implemented in a public college using 270 terminals and 9 servers. In this design, both staff and students can access their data from multiple locations equipped with thin clients and, as an added benefit, they are always presented with the same interface and settings. It was concluded in this study that a major benefit of this type of virtual setup was the reduction in power consumption of thin clients, which was approximately $1 / 10$ of the consumption that would be incurred by the full-fledged desktops or workstations. Therefore, applications of virtualization in campus environments reduce the cost of ownership and operation but also have significant reduction of their environmental impact. We expect, therefore, that the architecture of thin clients and servers will become widespread in educational institutions, thus further promoting the use of virtualization at the server side.

The use of virtual machines in the teaching of operating systems and OS administration was studied by Vollrath and Jenkins (2004). Teaching of operating systems requires at least one piece of full-fledged computer hardware for each student in the class. While using removable hard drives can be effective in minimizing the total number of computers needed, it is argued in (Vollrath and Jenkins, 2004) that virtual machine technology has the potential to not only reduce the operating costs of such laboratories but also simulate multiple computers on a single physical machine simultaneously. It is concluded that, while there are some drawbacks to using virtualization, the weight is on the advantages, meaning that virtualization has the potential to promote significantly the way we teach operating systems and system administration to date. In addition, virtualization environments can be replicated very easily in software and that allows the trainees for students to be focusing less on the installation of virtual machinery and more on management and the gist of the courses they are attending. 


\section{CONCLUSION}

Virtualization is a way of simultaneously running multiple operating systems on a physical computer. Virtualization can run on virtually every machine, from embedded systems-on-chip, to large datacenters and cloud environments. Simple on its surface, virtualization taps into possibilities that would be either unavailable or too involved to obtain from non-virtualized hardware.

Virtualization brings multiple benefits to the table: concurrent operation on non-native hardware; images of operating systems together with the associated data can be stored on networked systems and teleported; developers using virtualization can, at a fraction of the effort, develop a thorough grasp of the existing operating systems or work on their development, without the need to have all the diverse hardware actually deployed; the abundant computational power available today allows for real-time interaction with the virtual appliances; and the list goes on. At present, virtualization is a ripe technique supported both in the major CPU designs, and in the necessary virtualization software, and is already an inextricable part of production environments. With that, computer virtualization ought to become an inextricable part of the computer science (CS) education as well.

Together with the benefits of virtualization, there comes the burden of conquering this new technology by students, system administrators, and teachers at the tertiary level preparing the future CS experts. To be successful at these tasks, a wealth of information needs to be sifted through before selecting the right platforms for the computer science education and laboratory.

We provided a brief survey and analysis of virtualization and its applications, and traced the ways of its use in teaching of CS subjects. The hope is that this survey will be of value to instructors, laboratory administrators as well as to advanced students undertaking a self-study of operating systems and their applications.

\section{REFERENCE LIST}

Agrawal, S., Biswas, R., \& Nath, A. (2014). Virtual desktop infrastructure in higher education institution: Energy efficiency as an application of green computing. In Communication Systems and Network Technologies (CSNT), 2014 Fourth International Conference on, pages 601-605. IEEE.

Alabbadi, M. M. (2011). Cloud computing for education and learning: Education and learning as a service (elaas). In Interactive Collaborative Learning (ICL), 2011 14th International Conference on, pages 589-594. IEEE.

Alshwaier, A., Youssef, A., \& Emam, A. (2012). A new trend for e-learning in ksa using educational clouds. Advanced Computing, 3(1):81.

Anderson, B. R., Joines, A. K., \& Daniels, T. E. (2009). Xen worlds: leveraging virtualization in distance education. In ACM SIGCSE Bulletin, volume 41, pages 293-297. ACM.

Anisetti, M., Bellandi, V., Colombo, A., Cremonini, M., Damiani, E., Frati, F., Hounsou, J. T., \& Rebeccani, D. (2007). Learning computer networking on open paravirtual laboratories. IEEE Transactions on Education, 50(4):302-311.

Averitt, S., Bugaev, M., Peeler, A., Shaffer, H., Sills, E., Stein, S., Thompson, J., \& Vouk, M. (2007). Virtual computing laboratory ( $\mathrm{vcl})$. In Proceedings of the International Conference on the Virtual Computing Initiative, pages 1-6.

Bala, P. S. (2010). Intensification of educational cloud computing and crisis of data security in public clouds. International Journal on Computer Science and Engineering, 2(3):741-745.

Border, C. (2007). The development and deployment of a multi-user, remote access virtualization system for networking, security, and system administration classes. In ACM SIGCSE Bulletin, volume 39, pages 576-580. ACM.

Bower, T. (2010). Experiences with virtualization technology in education. Journal of Computing Sciences in Colleges, 25(5):311-318.

Cimen, C., Kavurucu, Y., \& Aydin, H. (2014). Usage of thin-client-server architecture in computer aided education. TOJET: The Turkish Online Journal of Educational Technology, 13(2). 
Davoli, R. (2005). Vde: Virtual distributed ethernet. In Testbeds and Research Infrastructures for the Development of Networks and Communities, (2005). Tridentcom (2005). First International Conference on, pages 213-220. IEEE.

Dawson, M., DeWalt, B., \& Cleveland, S. (2016). The case for ubuntu linux operating system performance and usability for use in higher education in a virtualized environment.

Dobrilovic, D. \& Stojanov, Z. (2006). Using virtualization software in operating systems course. In Information Technology: Research and Education, (2006). ITRE’06. International Conference on, pages 222-226. IEEE.

Egi, N., Greenhalgh, A., Handley, M., Hoerdt, M., Mathy, L., \& Schooley, T. (2007). Evaluating xen for router virtualization. In Computer Communications and Networks, (2007). ICCCN (2007). Proceedings of 16th International Conference on, pages 1256-1261. IEEE.

Ercan, T. (2010). Effective use of cloud computing in educational institutions. Procedia-Social and Behavioral Sciences, 2(2):938-942.

Fuertes, W., De Vergara, J. L., \& Meneses, F. (2009). Educational platform using virtualization technologies: Teaching-learning applications and research uses cases. In Proc. II ACE Seminar: Knowledge Construction in Online Collaborative Communities, volume 16.

Galán, F., Fernández, D., Ruiz, J., Walid, O., \& de Miguel, T. (2004). Use of virtualization tools in computer network laboratories. In Information Technology Based Higher Education and Training, (2004). ITHET (2004). Proceedings of the Flfth International Conference on, pages 209-214. IEEE.

Galán, F., Fernández, D., Fuertes, W., Gómez, M., \& de Vergara, J. E. L. (2009). Scenario-based virtual network infrastructure management in research and educational testbeds with vnuml. Annals of telecommunications-annales des télécommunications, 64(5-6):305-323.

Gaspar, A., Langevin, S., Armitage, W., \& Rideout, M. 2008a. Enabling new pedagogies in operating systems and networking courses with state of the art open source kernel and virtualization technologies. Journal of Computing Sciences in Colleges, 23(5):189-198.

Gaspar, A., Langevin, S., Armitage, W., Sekar, R., \& Daniels, T. 2008b. The role of virtualization in computing education. In ACM sIGCsE bulletin, volume 40, pages 131-132. ACM.

Gaspar, A., Langevin, S., Armitage, W. D., \& Rideout, M. 2008c. March of the (virtual) machines: past, present, and future milestones in the adoption of virtualization in computing education. Journal of Computing Sciences in Colleges, 23(5):123-132.

Huh, J.-H. \& Seo, K. (2016). Design and test bed experiments of server operation system using virtualization technology. Human-centric Computing and Information Sciences, 6(1):1.

Hwang, W.-Y., Kongcharoen, C., \& Ghinea, G. (2014). To enhance collaborative learning and practice network knowledge with a virtualization laboratory and online synchronous discussion. The International Review of Research in Open and Distributed Learning, 15(4).

Kalagiakos, P. \& Karampelas, P. (2011). Cloud computing learning. In Application of Information and Communication Technologies (AICT), 2011 5th International Conference on, pages 1-4. IEEE.

Khmelevsky, Y. \& Voytenko, V. (2010). Cloud computing infrastructure prototype for university education and research. In Proceedings of the 15th Western Canadian Conference on Computing Education, page 8. ACM.

Klement, M. (2017). Models of integration of virtualization in education: Virtualization technology and possibilities of its use in education. Computers \& Education, 105:31-43.

Kolyshkin, K. (2006). Virtualization in linux. White paper, OpenVZ, 3:39.

Laadan, O., Nieh, J., \& Viennot, N. (2010). Teaching operating systems using virtual appliances and distributed version control. In Proceedings of the 41st ACM technical symposium on Computer science education, pages 480-484. ACM.

Li, P. 2010a. Centralized and decentralized lab approaches based on different virtualization models. Journal of Computing Sciences in Colleges, 26(2):263-269.

Li, P. 2010b. Selecting and using virtualization solutions: our experiences with vmware and virtualbox. Journal of Computing Sciences in Colleges, 25(3):11-17. 
Lombardi, F. \& Di Pietro, R. (2011). Secure virtualization for cloud computing. Journal of Network and Computer Applications, 34(4):1113-1122.

Lunsford, D. L. (2009). Virtualization technologies in information systems education. Journal of Information Systems Education, 20(3):339.

Miller, K. \& Pegah, M. (2007). Virtualization: virtually at the desktop. In Proceedings of the 35th annual ACM SIGUCCS fall conference, pages 255-260. ACM.

Moritoh, Y. \& Imai, Y. (2015). Development of an integrated information server system for it education through server virtualization technology. IJNCAA, page 39.

Nieh, J. \& Vaill, C. (2005). Experiences teaching operating systems using virtual platforms and linux. In ACM SIGCSE Bulletin, volume 37, pages 520-524. ACM.

Pollitt, M., Nance, K., Hay, B., Dodge, R. C., Craiger, P., Burke, P., Marberry, C., \& Brubaker, B. (2008). Virtualization and digital forensics: A research and education agenda. Journal of Digital Forensic Practice, 2(2):62-73.

Rahimi, M. R., Ren, J., Liu, C. H., Vasilakos, A. V., \& Venkatasubramanian, N. (2014). Mobile cloud computing: A survey, state of art and future directions. Mobile Networks and Applications, 19(2):133143.

Romero-Zaldivar, V.-A., Pardo, A., Burgos, D., \& Kloos, C. D. (2012). Monitoring student progress using virtual appliances: A case study. Computers \& Education, 58(4):1058-1067.

Sayler, A., Grunwald, D., Black, J., White, E., \& Monaco, M. (2014). Supporting cs education via virtualization and packages: tools for successfully accommodating bring-your-own-device at scale. In Proceedings of the 45th ACM technical symposium on Computer science education, pages 313-318. ACM.

Stackpole, B., Koppe, J., Haskell, T., Guay, L., \& Pan, Y. (2008). Decentralized virtualization in systems administration education. In Proceedings of the 9th ACM SIGITE conference on Information technology education, pages 249-254. ACM.

Stewart, K. E., Humphries, J. W., \& Andel, T. R. (2009). Developing a virtualization platform for courses in networking, systems administration and cyber security education. In Proceedings of the 2009 spring simulation multiconference, page 65. Society for Computer Simulation International.

Sultan, N. (2010). Cloud computing for education: A new dawn? International Journal of Information Management, 30(2):109-116.

Vollrath, A. \& Jenkins, S. (2004). Using virtual machines for teaching system administration. Journal of Computing Sciences in Colleges, 20(2):287-292.

Wang, B. \& Xing, H. (2011). The application of cloud computing in education informatization. In Computer Science and Service System (CSSS), 2011 International Conference on, pages 2673-2676. IEEE.

Wang, X., Hembroff, G. C., \& Yedica, R. (2010). Using vmware vcenter lab manager in undergraduate education for system administration and network security. In Proceedings of the 2010 ACM conference on Information technology education, pages 43-52. ACM.

Wannous, M. \& Nakano, H. (2010). Nvlab, a networking virtual web-based laboratory that implements virtualization and virtual network computing technologies. IEEE Transactions on Learning Technologies, 3(2):129-138.

Xu, L., Huang, D., \& Tsai, W.-T. (2014). Cloud-based virtual laboratory for network security education. IEEE Transactions on Education, 57(3):145-150.

Zhao, H., Shi, L., \& Zhu, S. (2017). Application of virtualization technology in computer experiments. 